\title{
HYDROCARBONS, ICES, AND "XCN" IN THE LINE OF SIGHT TOWARD THE GALACTIC CENTER
}

\author{
J. E. Chiar, ${ }^{1}$ A. J. Adamson, ${ }^{2}$ Y. J. Pendleton, ${ }^{3}$ D. C. B. Whittet ${ }^{4}$ D. A. Caldwell ${ }^{3}$ And E. L. GibB ${ }^{4}$ \\ Received 2001 August 28; accepted 2002 January 13
}

\begin{abstract}
We discuss 2.8-3.9 $\mu \mathrm{m}$ spectra from the United Kingdom Infrared Telescope of seven sight lines toward IR sources near Sagittarius A* in the Galactic center (GC). In all lines of sight, the $3.0 \mu \mathrm{m} \mathrm{H}_{2} \mathrm{O}$ ice feature is present with optical depths in the range 0.33-1.52. By constructing a simple ice model, we show that the ice profile is not fully accounted for by pure $\mathrm{H}_{2} \mathrm{O}$ ice mantles. Residual absorption is present at 2.95 and 3.2-3.6 $\mu \mathrm{m}$. Aliphatic hydrocarbon absorption at $3.4 \mu \mathrm{m}$ is shown to vary by a factor of 1.7 , indicating significant changes in the foreground extinction across the small field. By determining the true ice profile for the GC line of sight, we reveal an additional broad absorption component around $\sim 3.3 \mu \mathrm{m}$, which partially underlies the $3.4 \mu \mathrm{m}$ aliphatic hydrocarbon feature. Its carrier resides in the diffuse interstellar medium. The width of this absorption is deduced to be at least $\sim 100 \mathrm{~cm}^{-1}$, much broader than individual polycyclic aromatic hydrocarbon molecules produced in the laboratory or unidentified infrared emission features observed in the interstellar medium. The $4.62 \mu \mathrm{m}$ "XCN" feature is detected in the molecular clouds along the line of sight toward IRS 19. In the solar neighborhood, this feature is seen only toward some deeply embedded protostars. Toward the GC, it may indicate the serendipitous presence of such an object in the line of sight to IRS 19, or it might conceivably arise from the processing of ices in the circumnuclear ring of the GC itself.
\end{abstract}

Subject headings: dust, extinction — Galaxy: center — infrared: ISM: lines and bands — infrared: stars — ISM: molecules

\section{INTRODUCTION}

The abundance and nature of ices in the interstellar medium are of long-standing interest. Their potential power as diagnostics of the physical and evolutionary state of a molecular cloud region are well known, and the accretion of interstellar ices onto young planets via interplanetary dust particles and comets may be a key to the formation of atmospheres, oceans, and the production of prebiotic molecules. Processed ices likely contribute some proportion of the more refractory grain materials that persist when the molecular cloud is dispersed by star formation, and these products may yield the extinction characteristic of the diffuse interstellar medium. Furthermore, the depletion of gas-phase species onto grain surfaces significantly affects gas-phase chemistry in dense molecular clouds and the abundance of molecular hydrogen inferred from tracers such as CO.

The line of sight toward the Galactic center (GC) is dominated by diffuse interstellar medium (ISM) dust (Lebofsky 1979); however, it is now clear that molecular cloud material also contributes to the $30 \mathrm{mag}$ of visual extinction (Gerakines et al. 1999; de Graauw et al. 1996; Lutz et al. 1996). Much of the molecular cloud extinction presumably arises in dust clouds located within $4 \mathrm{kpc}$ of Earth (Sanders, Scoville, \& Solomon 1985). If this is so, then the clouds are not associated with the infrared sources that provide the

\footnotetext{
${ }^{1}$ NASA-Ames Research Center, Mail Stop 245-3, Moffett Field, CA 94035; and SETI Institute, 2035 Landings Drive, Mountain View, CA 94043 .

2 Joint Astronomy Centre, 660 North A'ohoku Place, Hilo, HI 96720.

${ }^{3}$ NASA Ames Research Center, Mail Stop 245-3, Moffett Field, CA 94035 .

${ }^{4}$ Department of Physics, Applied Physics and Astronomy, Rensselaer Polytechnic Institute, Troy, NY 12180.
}

continuum against which molecular absorption features can be observed. Recent measurements of infrared and submillimeter $\mathrm{CO}$ and $\mathrm{H}_{2} \mathrm{O}$ lines have shown that the absorbing gas along the GC line of sight is cold $(\sim 10 \mathrm{~K})$ and that most of the $\mathrm{H}_{2} \mathrm{O}$ has condensed onto grains (Moneti, Cernicharo, \& Pardo 2001a). The molecular clouds along the line of sight toward the GC are similar to local molecular clouds (within $1 \mathrm{kpc}$ of the Sun) in terms of abundances of key solid state molecules (Chiar et al. 2000). Water ice remains the most abundant solid-state molecule; the solid $\mathrm{CO}_{2} / \mathrm{H}_{2} \mathrm{O}$ ratio is within the range found for local molecular clouds (Gerakines et al. 1999); $\mathrm{CH}_{3} \mathrm{OH} / \mathrm{H}_{2} \mathrm{O}$ is similar to that observed in quiescent clouds such as Taurus (Chiar et al. 1996) and does not resemble the higher abundance observed toward heavily embedded massive protostars (Grim et al. 1991; Allamandola et al. 1992; Dartois et al. 1999). Solid CO was weakly detected toward one IR source; however, gas-phase $\mathrm{CO}$ seems to dominate the absorption around $4.67 \mu \mathrm{m}$ toward the Sgr A* region (Moneti et al. 2001; Chiar et al. 2000; McFadzean et al. 1989). The implied solid CO/ $\mathrm{H}_{2} \mathrm{O}$ ratio is less than $10 \%$. The $3.0 \mu \mathrm{m}$ ice profile observed toward the GC is distinct from that seen in local molecular clouds such as Taurus (McFadzean et al. 1989; Tielens et al. 1996; Chiar et al. 2000). It is consistent with cold ( 15 K) ices, similar to local quiescent dense clouds, but peaks at $\sim 2.96 \mu \mathrm{m}$, shortward of the local molecular cloud ice feature. This might be interpreted as evidence for enhanced $\mathrm{NH}_{3}$ abundance in the ices toward the GC (Chiar et al. 2000).

Infrared sources located in the region near the radio source $\mathrm{Sgr} \mathrm{A}^{*}$ have been used extensively to characterize organic grain material in the diffuse interstellar medium (Butchart et al. 1986; Sandford et al. 1991). Aliphatic hydrocarbon material is characterized by its $\mathrm{CH}_{2}$ (methylene) and $\mathrm{CH}_{3}$ (methyl) stretching modes around $3.4 \mu \mathrm{m}$ (Sandford et al. 1991; Pendleton et al. 1994). These hydrocarbons are 
most likely carried by a population of very small unaligned grains, rather than refractory mantles on silicate cores (Adamson et al. 1999; Chiar et al. 1998). Aromatic hydrocarbons are detected via their $\mathrm{CH}$ and $\mathrm{CC}$ stretching modes at 3.28 and $6.2 \mu \mathrm{m}$, respectively, along the line of sight toward the Quintuplet region, $30 \mathrm{pc}$ away (in projection) from Sgr A*. Both absorption features appear to be much weaker toward Sgr A* compared with those toward the Quintuplet sources (Chiar et al. 2000). However, an absorption feature at $3.28 \mu \mathrm{m}\left(\mathrm{FWHM} \sim 50 \mathrm{~cm}^{-1}\right)$ was tentatively detected toward IRS 3, 6E, and 7 by Pendleton et al. (1994). They suggested that $\sim 5 \%$ of all the carbon along these lines of sight could be tied up in aromatic hydrocarbons attached to hydrogen atoms. Chiar \& Tielens (2001) suggest that the $6.2 \mu \mathrm{m}$ absorption feature is actually produced by late-type dusty carbon-rich Wolf-Rayet (WC) stars and is not interstellar in nature. Their results support the suggestion that the Quintuplet sources are late-type WC stars and imply that the 3.28 and $6.2 \mu \mathrm{m}$ features would have independent carriers. An absorption feature at $3.25 \mu \mathrm{m}$ is detected in ground-based data in five heavily extincted molecular cloud sight lines and is attributed to aromatic hydrocarbon molecules at low temperature (Sellgren et al. 1995; Brooke et al. 1999). Differences in the central wavelength and width of the $3.3 \mu \mathrm{m}$ profiles can be attributed to differences in temperature and/or carrier of the aromatic materials in these regions.

The main aims of this paper are (1) to trace the spatial distribution of dense and diffuse ISM dust across the Sgr A* field, and (2) to disentangle absorption features due to diffuse ISM and dense cloud material along the line of sight. New 2.8-3.9 $\mu \mathrm{m}$ data for seven sources, described in $\S 2$, were obtained with the CGS4 spectrometer on the United Kingdom Infrared Telescope (UKIRT); their spectra are presented in $\S 3$. In $\S 4$, we describe our deconvolution of ice and hydrocarbon absorption features in the $3 \mu \mathrm{m}$ region. Sections 5 and 6 discuss the nature of molecular cloud ices $\left(\mathrm{H}_{2} \mathrm{O}, \mathrm{CO}\right.$, "XCN") and hydrocarbon material, respectively, along the line of sight. Finally, we summarize our results in $\S 7$.

\section{GENERAL MORPHOLOGY AND NATURE OF THE OBJECTS}

The Galactic center central cavity is about 3 pc in diameter (assuming a distance of $8.5 \mathrm{kpc}$ ). It is surrounded by a higher density ring of gas and dust, referred to as the circumnuclear ring, which is inclined $60^{\circ}-70^{\circ}$ with respect to the line of sight (Güsten et al. 1987). Interferometric HCN observations show that the extinction within the ring is clumpy, and IR observations show that the extinction across the central cavity is also nonuniform (Chan et al. 1997). Visual extinction estimates range between 29 and 37 mag for the sources discussed here (see Rieke, Rieke, \& Paul 1989 for a review of extinction estimates). The extinction consists of general diffuse ISM dust as well as dense cloud material. An attempt to decouple the contribution of each of these reservoirs of interstellar material is presented in $\S 4$.

The infrared sources, IRS 1, 3, 6, 7, 8, 12, and 19, were discovered by the infrared surveys of Reike \& Low (1973) and Becklin \& Neugebauer (1975). IRS 1 was later resolved into multiple components by Storey \& Allen (1983), Rieke et al. (1989), Simon et al. (1990), and Herbst et al. (1993). Tollestrup et al. (1989) resolved IRS 6 and IRS 12 into mul-

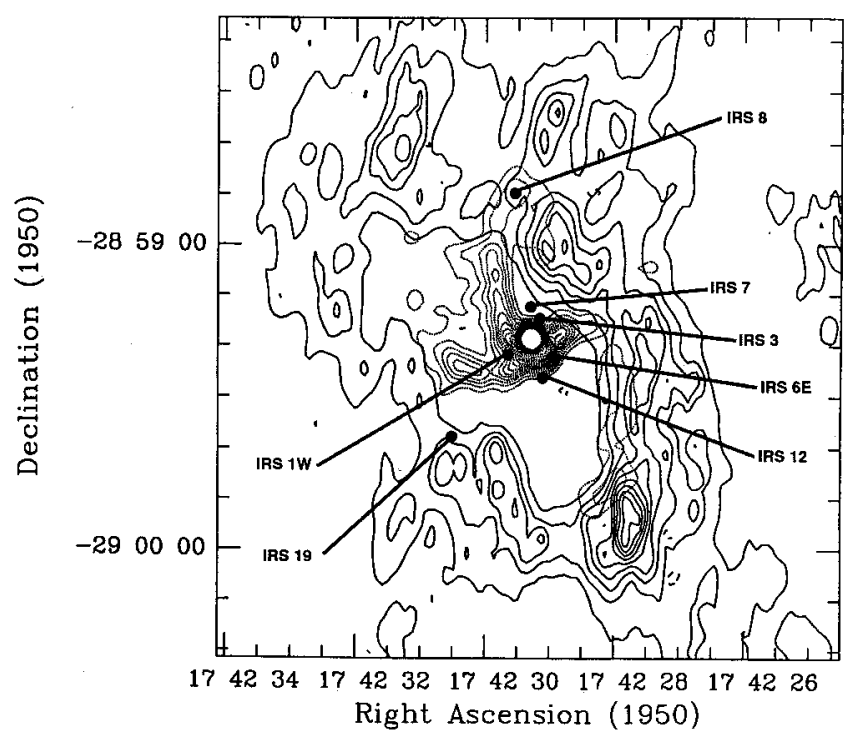

FIG. 1.-Positions of infrared sources overlaid on a map of $\mathrm{HCN}$ $J=1-0$ emission (solid contours) and ionized gas at $15 \mathrm{GHz}$ (dotted contours) (reproduced from Güsten et al. 1987). Contour interval for the velocity-integrated $\mathrm{HCN}$ emission is $0.15 \mathrm{~K}$ averaged over $300 \mathrm{~km} \mathrm{~s}^{-1}$ in a $2^{\prime \prime}$ beam. Dotted contours of $15 \mathrm{GHz}$ emission at $20 \mathrm{~K}$ intervals in a 3 .' $6 \times 3$ ". 4 beam. The radio source $\mathrm{Sgr} \mathrm{A}^{*}$ is located at $\alpha=17^{\mathrm{h}} 42^{\mathrm{m}} 29^{\mathrm{s}} .2$ $\delta=-28^{\circ} 59^{\prime} 19^{\prime \prime}$. The GC Quintuplet sources are located $14^{\prime}$ northeast of the $\mathrm{GC}, \sim 30 \mathrm{pc}$ away (assuming a distance of $8.5 \mathrm{kpc}$ ).

tiple components. The GC infrared sources discussed in this paper are located within a projected distance of $2 \mathrm{pc}$ of Sgr $A^{*}$ (Fig. 1). IRS 8 and IRS 19 are $30^{\prime \prime}$ north and southwest, respectively, from the central cluster (which includes IRS 1, $3,6,7$, and 12); their lines of sight intersect the circumnuclear ring (Fig. 1; Geballe, Baas, \& Wade 1989; Güsten et al. 1987). Of the sources discussed here, IRS $1,3,6$, and 8 are strong $10 \mu \mathrm{m}$ sources, whereas IRS 7, 12, and 19 are brightest at $2 \mu \mathrm{m}$ (Becklin et al. 1978; Rieke \& Low 1973). The latter are $\mathrm{M}$ (super)giants (IRS 19 [M4 Ib], IRS $12 \mathrm{~N}$ [M7 III], and IRS 7 [M1 I]) (Wollman, Smith, \& Larson 1982; Lebofsky, Rieke, \& Tokunaga 1982; Blum, Sellgren, \& Depoy 1996). The nature of IRS 8 and IRS 3 is unknown; Becklin et al. (1978) suggest that IRS 3 is an OH/IR star, although it has a nearly featureless $K$-band spectrum (Blum et al. 1996). IRS $1 \mathrm{~W}$ is the brightest component (of IRS 1 ) at $L(3.5 \mu \mathrm{m})$. This source shows no He I emission and is most likely an $\mathrm{H}$ II region with an embedded source (Blum, Depoy, \& Sellgren 1995 and references therein). IRS 6E is the brightest at $L$ of two components that make up IRS 6 (Tollestrup et al. 1989) and has been identified as a late-type WR star (WC9) by Krabbe et al. (1995).

\section{OBSERVATIONS AND DATA REDUCTION}

The Cooled Grating Spectrometer (CGS4) on UKIRT was used to acquire data in the $2.8-3.9 \mu \mathrm{m}$ spectral region of the Galactic center sources IRS 1W, 3, 6E, 8, 12N, and 19 on 1998 July 11-14. The positions of these sources superposed on an HCN map (reproduced from Güsten et al. 1987) are shown in Figure 1. In order to point the telescope accurately on the sources in the central cluster, IRS 7 was used as a reference point. We "peaked-up" (centered the source in the slit) here, then offset the telescope to the desired source using the positional information from Bailey, 
TABLE 1

Observing Parameters, Photometry, and Parameters Used in Blackbody Fit

\begin{tabular}{|c|c|c|c|c|c|c|c|c|}
\hline \multirow[b]{2}{*}{ SOURCE } & \multirow[b]{2}{*}{ WAVELENGTH $^{\mathrm{a}}$} & \multirow[b]{2}{*}{ Position ANGLE ${ }^{\mathrm{b}}$} & \multirow[b]{2}{*}{$K(2.22 \mu \mathrm{M})$} & \multirow[b]{2}{*}{ REFERENCES } & \multirow[b]{2}{*}{$L(3.45 \mu \mathrm{M})$} & \multirow[b]{2}{*}{ REFERENCES } & \multicolumn{2}{|c|}{ BLACKBODY PARAMETERS } \\
\hline & & & & & & & $T$ & $A_{K}$ \\
\hline IRS $1 \mathrm{~W} \ldots \ldots \ldots . .$. & $3.11,3.60$ & 90,90 & 8.7 & 1,2 & 5.5 & 1,2 & 1100 & 3.10 \\
\hline IRS $3 \ldots \ldots \ldots \ldots$ & $3.11,3.60$ & 90,148 & 10.98 & 1 & 5.2 & 1,2 & 1200 & 3.00 \\
\hline IRS 6E ........... & $3.11,3.60$ & 90,90 & 9.93 & 1 & 6.3 & 2 & 1300 & 4.00 \\
\hline IRS $7^{\mathrm{c}, \mathrm{d}} \ldots \ldots \ldots$ & $3.15,3.55$ & 180,180 & 6.7 & 3 & 4.5 & 3 & 3400 & 3.90 \\
\hline IRS $8 \ldots \ldots \ldots \ldots$ & $3.11,3.60$ & 90,90 & 10.3 & 1,3 & 6.1 & 3 & 1000 & 4.00 \\
\hline IRS $12 \mathrm{~N}^{\mathrm{d}} \ldots \ldots$ & $3.11,3.60$ & 126,126 & 8.1 & 3 & 5.7 & 3 & 1000 & 3.15 \\
\hline \multirow[t]{2}{*}{ IRS $19 \ldots \ldots \ldots \ldots$} & $3.11,3.60$ & 90,90 & 8.2 & 1 & 6.5 & 3 & 3400 & 3.50 \\
\hline & 4.80 & 90 & $\ldots$ & $\ldots$ & $\ldots$ & $\ldots$ & $\ldots$ & $\ldots$ \\
\hline
\end{tabular}

Hough, \& Axon (1984) and Tollestrup et al. (1989). A $256 \times 256 \mathrm{InSb}$ array was used with the 40 lines $\mathrm{mm}^{-1}$ grating and 1 pixel slit width, corresponding to 0.6 a mean resolving power $(\lambda / \Delta \lambda)$ of $R \sim 1200$. A $4.5-5 \mu \mathrm{m}$ spectrum was obtained with the 2 pixel slit, yielding a mean resolving power of $R \sim 900$. The position angles are listed in Table 1 . Where the position angle equals $90^{\circ}$, a sky position $5^{\prime}$ north of the program star was used for sky subtraction. For position angles other than $90^{\circ}$, nodding along the slit was employed to achieve sky subtraction. Observations of the standard star HR 6616 (F7II) at similar air mass were used to cancel telluric features in our program stars.

In addition, data from the UKIRT/CGS4 archive at the UK Astronomy Data Centre in Cambridge were obtained for IRS 7. These data were originally obtained 1995 July 12 with the $256 \times 256 \mathrm{InSb}$ array and the 75 lines $\mathrm{mm}^{-1}$ grating and 1 pixel slit. The standard star used was HD 154368 (O9 Ia).

Wavelength calibration for all observations was achieved using an argon lamp in second order. Flux calibration was achieved by ratioing each program star spectrum with that of the standard star, multiplying by a blackbody $(T=6150$ $\mathrm{K}$ for HR 6616; $T=30,000 \mathrm{~K}$ for HD 154368), and then normalizing the resulting average flux in the $L$ band (3.15$3.75 \mu \mathrm{m}$ ) to photometry from the literature (see Table 1). The flux-calibrated spectra are presented in Figure 2. Emission features due to recombination lines of atomic hydrogen are present at 3.04, 3.30, and $3.74 \mu \mathrm{m}$ in the spectra of IRS $1 \mathrm{~W}, 3,6 \mathrm{E}, 8$, and $12 \mathrm{~N}$ and are weakly present in IRS 19.

\section{DETERMINATION OF OPTICAL DEPTH SPECTRA}

We have chosen to use reddened blackbody curves to fit our spectra in a consistent way with a minimum of free parameters. Ground-based $K$-band photometry from the literature (Table 1) was used to constrain our fits on the short-wavelength side, since telluric absorption shortward of $2.8 \mu \mathrm{m}$ prevents observation of the blue side of the ice profile from the ground. Blackbody curves were computed by using the universal extinction curve for dense and diffuse clouds determined by Martin \& Whittet (1990) and restricting the $K$-band extinction to the average range $\left(A_{K}=2-4\right.$ mag) found for the central $2^{\prime}$ of the Galaxy (Blum et al.
1996). We matched the reddened blackbodies using the $K$-band photometry point and spectral data from $\lambda>3.75$ $\mu \mathrm{m}$ to constrain the blackbody parameters. The temperature was allowed to vary within constraints set by the nature of the sources $(\S 2)$. The results of the blackbody fits are listed in Table 1. The late-type objects (IRS 7 and IRS 19; we return to IRS $12 \mathrm{~N}$ later) were fitted with temperatures constrained to around $3300 \mathrm{~K}$. Sources with large $10 \mu \mathrm{m}$ excesses (IRS 1W, 3, 6E, and 8) were fitted with much lower temperatures (of the order of $1000 \mathrm{~K}$ ). In all these cases, the observed long-wavelength continuum is smoothly joined by the fitted blackbody. In the case of IRS $12 \mathrm{~N}$, attempts to fit the continuum with $T \sim 3000 \mathrm{~K}$ blackbodies caused a discontinuity in slope at the long-wavelength end unless implausibly large values of the extinction $\left(A_{K} \gg 4\right)$ were adopted. For this one object, therefore, the temperature was allowed to vary outside that formally expected from the spectral type; a final value of $1100 \mathrm{~K}$ was adopted, with a $K$ band extinction of $3.15 \mathrm{mag}$. Even these values give a less than convincing fit, and the optical depth spectrum of this object remains uncertain. The blackbody fits and the resulting optical depth spectra are shown in Figures 2 and 3, respectively.

\subsection{Determination of Absorption Feature Profiles}

The wide variation in the relative strength of ice and hydrocarbon absorptions in these lines of sight has been known for some years. Figure 3 shows that the ice band is strongly variable and that the hydrocarbon absorption around $3.4 \mu \mathrm{m}$ is less so (compare the profiles of IRS 19 and IRS $1 \mathrm{~W}$, and see Table 2). To further quantify the properties of these features, we attempt to isolate the two bands spectrally by considering variations between the sources, rather than by making a priori assumptions about the band profiles. The ice band was isolated first. The spectra were normalized to their mean value in a window near the peak of the band (3.0-3.03 $\mu \mathrm{m})$. Inspection of the results shows that the spectra of these sources have just two distinct components (Fig. 4). The ice band is of essentially constant profile, and there is a hydrocarbon component that becomes distinct from the ice band at $3.15 \mu \mathrm{m}$ and extends to beyond $3.7 \mu \mathrm{m}$. 


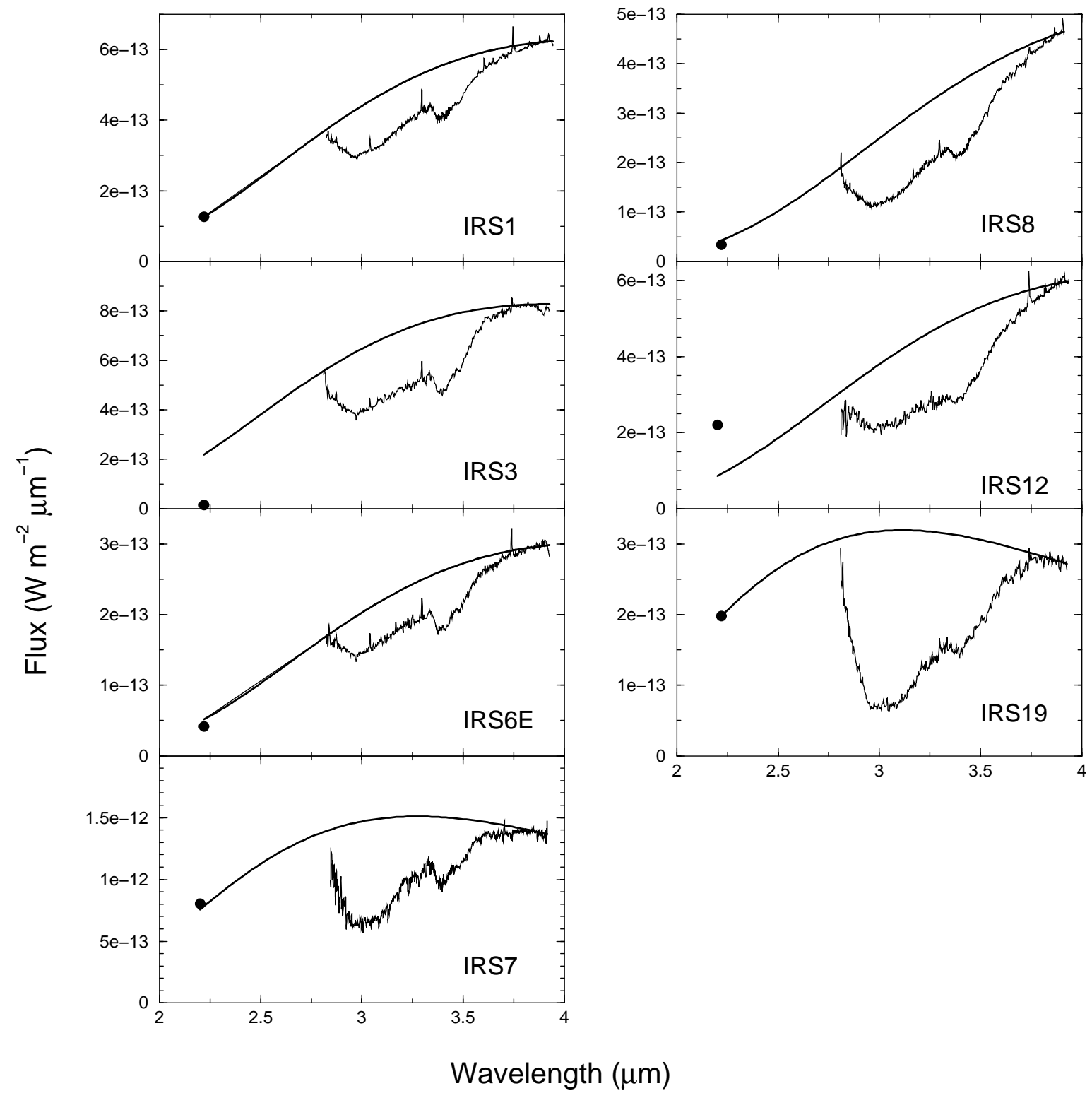

FIG. 2.-Flux spectra of the GC IR sources. The spectra have been scaled to agree with $L$-band photometry from the literature (see Table 1). Blackbody curves (smooth lines), normalized to the astronomical spectra longward of $3.75 \mu \mathrm{m}$, are used to determine the optical depth spectra shown in Fig. 3 . $K$-band photometry from the literature (see Table 1) is also shown ( filled circles).

The spectral profile of the hydrocarbon absorption appears to vary little from source to source, although in the normalized spectra (Fig. 4) its intensity relative to the ice band is clearly strongly variable. In principle, the profile of this distinct band can be obtained by subtracting any pair of the normalized spectra, thus canceling the normalized (and constant) ice profile and leaving some constant multiple of the hydrocarbon profile. A better signal-to-noise ratio can clearly be obtained by carrying out a similar process for more than one pair. The spectrum of IRS 19 (which has the weakest $3.4 \mu \mathrm{m}$ absorption relative to the ice band) was therefore subtracted from every other spectrum (apart from IRS $12 \mathrm{~N}$, which is noisy and would be unsafe to include in the mean). An average "residual" spectrum, representing the hydrocarbon absorption, was then calculated.

Once the spectrum of the hydrocarbon component is known, the mean ice profile for the GC molecular clouds can be determined (Fig. 5). This was done by interactively subtracting a variable proportion of the average hydrocarbon spectrum from each individual spectrum (as normalized at $3.0 \mu \mathrm{m}$ previously) until the spectrum in the wavelength range of the hydrocarbon contamination was featureless. With two exceptions, this was straightforward and indeed resulted in a smooth ice profile, further strengthening the case that the shape of the hydrocarbon band is invariant. The exceptions were IRS $12 \mathrm{~N}$ and IRS 7 . The former is very noisy, as mentioned above, and was excluded from the calculated mean. The latter appears to have an anomalously narrow ice band profile.

IRS 19 has a late-type spectrum contaminated by narrow photospheric $\mathrm{OH}$ features, which propagate into the subtracted spectra and hence into the final derived hydrocarbon band. It is therefore possible to further improve on the hydrocarbon spectrum by one additional iteration, this time 


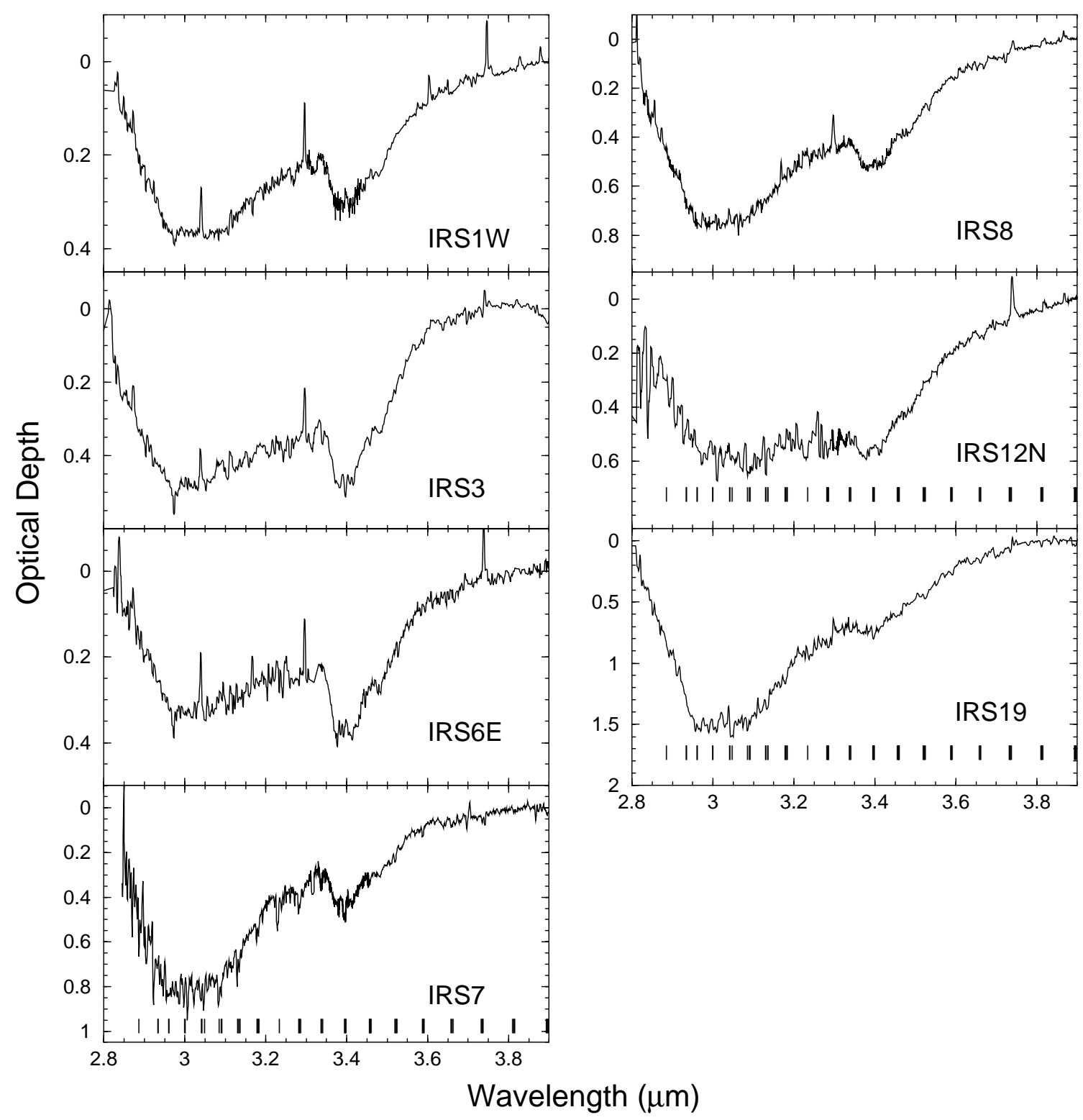

FIG. 3.-Optical depth spectra of the GC IR sources determined by ratioing with the blackbody curves shown in Fig. 1. The vertical bars on the plots of the known M stars (IRS 7, IRS 12N, and IRS 19) denote the positions of OH lines (taken from Hinkle, Wallace, \& Livingston 1995).

TABLE 2

Optical Depths and Column Densities of Dust Absorption Features

\begin{tabular}{|c|c|c|c|c|c|c|c|c|}
\hline Source & $\tau_{3.0 \mu \mathrm{m}^{\mathrm{a}}}$ & $\tau_{3.3 \mu \mathrm{m}^{\mathrm{b}}}$ & $\tau_{3.4 \mu \mathrm{m}^{\mathrm{b}}}$ & $\tau_{4.62 \mu \mathrm{m}}$ & $\tau_{4.67 \mu \mathrm{m}}$ & $\begin{array}{c}N\left(\mathrm{H}_{2} \mathrm{O}\right) \\
\times 10^{17} \mathrm{~cm}^{-2}\end{array}$ & $\begin{array}{c}N(\mathrm{CO}) \\
\times 10^{17} \mathrm{~cm}^{-2}\end{array}$ & $\begin{array}{c}N(\mathrm{XCN}) \\
\times 10^{17} \mathrm{~cm}^{-2}\end{array}$ \\
\hline IRS $1 \mathrm{~W} \ldots$. & $0.37 \pm 0.02$ & $0.052 \pm 0.003$ & $0.179 \pm 0.003$ & $\ldots$ & $\ldots$ & $8.7 \pm 0.5$ & $\ldots$ & $\ldots$ \\
\hline IRS $3 \ldots \ldots \ldots$ & $0.48 \pm 0.03$ & $0.124 \pm 0.004$ & $0.310 \pm 0.004$ & $\ldots$ & $\ldots$ & $11.1 \pm 0.5$ & $\ldots$ & $\ldots$ \\
\hline IRS 6E ..... & $0.33 \pm 0.05$ & $0.076 \pm 0.006$ & $0.259 \pm 0.004$ & $\ldots$ & $\ldots$ & $7.5 \pm 0.5$ & $\ldots$ & $\ldots$ \\
\hline IRS 7 .......... & $0.8 \pm 0.1$ & $-0.024 \pm 0.011$ & $0.147 \pm 0.005$ & $\ldots$ & $\ldots$ & $18 \pm 1$ & $\ldots$ & $\ldots$ \\
\hline IRS $8 \ldots \ldots \ldots$ & $0.75 \pm 0.04$ & $0.080 \pm 0.005$ & $0.258 \pm 0.003$ & $\ldots$ & $\ldots$ & $17.0 \pm 0.5$ & $\ldots$ & $\ldots$ \\
\hline IRS $12 \mathrm{~N} \ldots$ & $0.6 \pm 0.2$ & $0.216 \pm 0.014$ & $0.357 \pm 0.003$ & $\ldots$ & $0.15 \pm 0.05^{\mathrm{c}}$ & $14 \pm 1$ & $\ldots$ & $\ldots$ \\
\hline IRS $19 \ldots \ldots$ & $1.52 \pm 0.03$ & $0.082 \pm 0.009$ & $0.222 \pm 0.007$ & $0.6 \pm 0.1$ & $0.7 \pm 0.2$ & $36 \pm 4$ & $4 \pm 1$ & $3.0 \pm 0.5$ \\
\hline
\end{tabular}

a Uncertainties listed are limits on the allowable depth of the feature based on uncertainties in the continuum fitting.

b Optical depths for 3.3 and $3.4 \mu \mathrm{m}$ features are deduced by subtracting a mean ice profile from each spectrum. Since the ice profile for IRS 7 is more narrow than the mean, a negative value for $\tau_{3.3}$ results. See text for details.

c Optical depth for solid CO from McFadzean et al. 1989. 


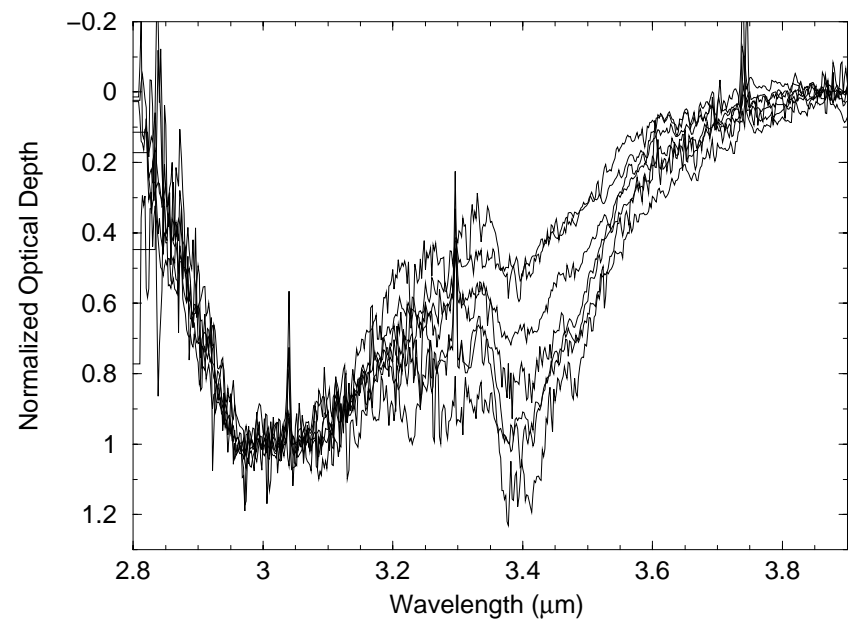

FIG. 4. - Optical depth spectra of the GC IR sources normalized at the peak of the ice profile between 3.00 and $3.05 \mu \mathrm{m}$ to $\tau=1$. This figure shows that the ice profiles for the GC IR sources are similar between 2.8 and 3.1 $\mu \mathrm{m}$. The profiles begin to deviate longward of $3.1 \mu \mathrm{m}$, where absorption due to hydrocarbons dominates the spectrum (see text).

replacing the IRS 19 spectrum, which was selected because it had a relatively weak $3.4 \mu \mathrm{m}$ band, with a smoothed version of the mean variable ice band. This produces the mean residual spectrum shown in Figure 6.

\section{MOLECULAR CLOUD ICES}

Molecular cloud ices along the GC line of sight are characterized by absorption features due to $\mathrm{H}_{2} \mathrm{O}$ (3.0 and 6.0 $\mu \mathrm{m}), \mathrm{CO}_{2}(4.27$ and $15.3 \mu \mathrm{m})$, and $\mathrm{CH}_{4}(7.67 \mu \mathrm{m})$ (Chiar et al. 2000; Gerakines et al. 1999; de Graauw et al. 1996; Lutz et al. 1996). In the following sections, we discuss the $3.0 \mu \mathrm{m}$ $\mathrm{H}_{2} \mathrm{O}$ ice profile and the 4-5 $\mu$ m spectrum of IRS 19, which exhibits both the solid $\mathrm{CO}(4.67 \mu \mathrm{m})$ and XCN $(4.62 \mu \mathrm{m})$ features.

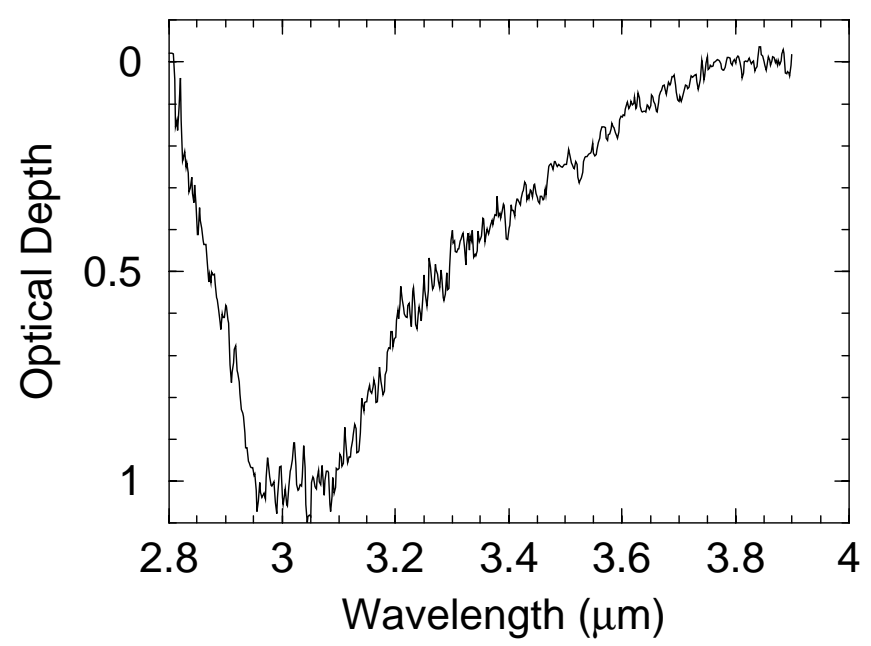

FIG. 5.-Mean ice spectrum with hydrocarbon absorption removed as described in text ( $\$ 4.1)$. IRS $12 \mathrm{~N}$ and IRS 7 are excluded from the mean.

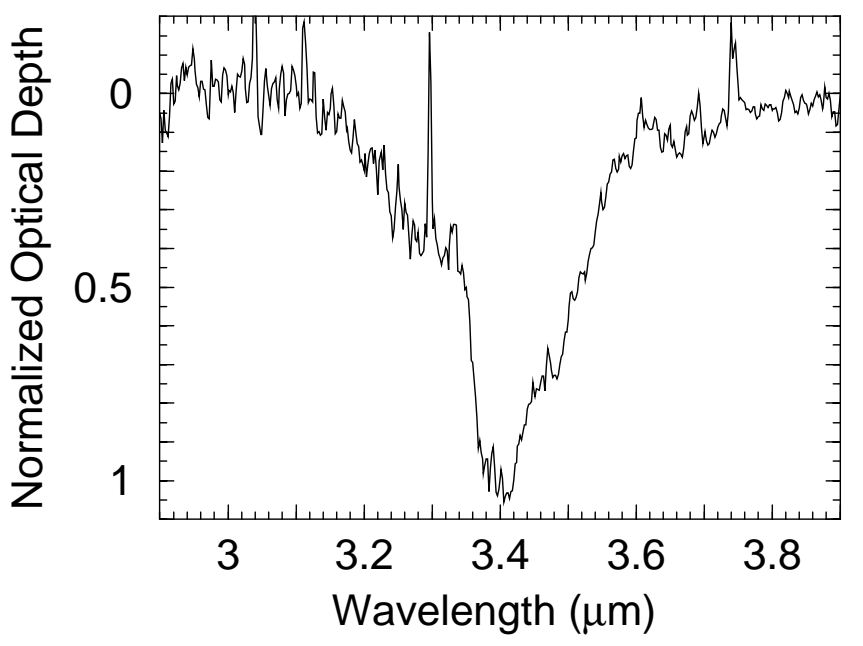

FIG. 6.-Mean residual (hydrocarbon) spectrum deduced by subtracting the mean ice profile (Fig. 5) from IRS 1W, 3, 6E, 8, and 19. Absorption centered at $3.4 \mu \mathrm{m}$ is due to aliphatic hydrocarbons, whereas absorption centered around $3.3 \mu \mathrm{m}$ may be due to aromatic hydrocarbons.

\subsection{The $3.0 \mu \mathrm{m} \mathrm{H}_{2} \mathrm{O}$ Ice Profile}

The $\mathrm{H}_{2} \mathrm{O}$ ice profile shape is remarkably consistent across the seven sight lines studied here, including IRS 8 and IRS 19 , which are located apart from the central cluster, closer to the GC circumnuclear ring (Fig. 1; Geballe et al. 1989 and references therein; Latvakoski et al. 1999). The variation of the $3.0 \mu \mathrm{m}$ profile depth across the central GC field has been noted previously by McFadzean et al. (1989). Compared to local molecular cloud ice profiles, e.g., in Taurus, the GC profile is broader, peaks at shorter wavelengths, and has a deeper long-wavelength wing. Laboratory spectra of pure $\mathrm{H}_{2} \mathrm{O}$ ice do not fit the $\mathrm{GC}$ ice profile; additional absorption is present around $2.95 \mu \mathrm{m}$ and longward of 3.1 $\mu \mathrm{m}$ (in addition to the hydrocarbon absorption band described in $\S 6$ ). Previous work suggested that the $3.0 \mu \mathrm{m}$ feature along with its $6 \mu \mathrm{m}$ counterpart was due to $\mathrm{H}_{2} \mathrm{O}$ trapped in an $\mathrm{SiO}$ condensate (Wada et al. 1991; Tielens et al. 1996). However, nondetection of the corresponding 2.75 $\mu \mathrm{m}$ feature (Whittet et al. 1997) and the unambiguous detection of other ices along the line of sight now make that an unsuitable candidate. Although crystalline water shows substructure around $2.95 \mu \mathrm{m}$ (e.g., Smith, Sellgren, \& Tokunaga 1989; Hudgins et al. 1993), the profile peaks at longer wavelengths $(\sim 3.1 \mu \mathrm{m})$ than the observed GC feature. Chiar et al. (2000), by carrying out a simultaneous fit to the 3 and $6 \mu \mathrm{m}$ ice profiles, explain the unique profile by a high abundance of $\mathrm{NH}_{3}\left(\sim 20 \%\right.$ relative to $\left.\mathrm{H}_{2} \mathrm{O}\right)$ in the ices. Dartois \& d'Hendecourt (2001) require less $\mathrm{NH}_{3}$ in their models, which explain the $3 \mu \mathrm{m}$ ice profile in terms of scattering effects due to submicron-sized grains. Their fits imply $11 \%-$ $17 \%$ ammonia in the ices along the GC sight line. Finally, Gibb, Whittet, \& Chiar (2001) limit the $\mathrm{NH}_{3}$ abundance to less than 5\% toward Sgr A* based on the lack of a distinct 9 $\mu \mathrm{m} \mathrm{NH} \mathrm{NH}_{3}$ absorption feature in the deep $10 \mu \mathrm{m}$ silicate band. However, this study is based on Infrared Space Observatory (ISO) SWS data (centered on IRS 7) taken with a $14^{\prime \prime} \times 20^{\prime \prime}$ aperture. This complicates the analysis of the silicate profile, which is due to both emission and absorption of silicates in the field. Consequently, it is difficult to rule out the possible presence of an ammonia feature at $9 \mu \mathrm{m}$. 


\subsubsection{Modeling the Ice Absorption Profile}

To further elucidate the nature of the icy mantles in the molecular clouds along the GC line of sight, we modeled the $3 \mu \mathrm{m}$ ice profile in terms of simple core-mantle grains. The model presented here is meant to give a general overview of the $3 \mu \mathrm{m}$ profile shape compared with those for local dense clouds, such as Taurus, which have been modeled by similar methods. A more complete model is beyond the scope of this paper. Our model is based on the core-mantle Mie scattering code developed by Bohren \& Huffman (1983). Following the classic interstellar dust model of Mathis, Rumpl, \& Nordsieck (1977; hereafter MRN77), we assumed that the grain cores are composed of equal numbers of silicate and graphite grains and that the number density of grains follows the size distribution

$$
n\left(a_{c}\right) d a_{c}=A n_{\mathrm{H}} a_{c}^{-3.5} d a_{c},
$$

where $n_{\mathrm{H}}$ is the number density of $\mathrm{H}$ nuclei and $a_{c}$ is the core size, ranging from $0.005<a_{c}(\mu \mathrm{m})<0.25$. While the MRN77 model is based on modeling the extinction in diffuse clouds, this model should be a reasonable first approximation at the grain size distribution in dense clouds (e.g., Smith et al. 1993; Whittet et al. 1996; Holbrook \& Temi 1998). Based on the best fit to the average observed interstellar extinction curve, Draine \& Lee (1984) determined the constant $A$ to be $A$ (silicate $)=10^{-25.11} \mathrm{~cm}^{2.5} \mathrm{H}^{-1}$ and $A$ (graphite $)=10^{-25.16} \mathrm{~cm}^{2.5} \mathrm{H}^{-1}$. We used optical constants for "astronomical silicates" and graphite as computed by Weingartner \& Draine (2001), Laor \& Draine (1993), and Draine \& Lee (1984).

As a consequence of the MRN77 grain size distribution (eq. [1]), small grains numerically outnumber large grains. Thus, assuming a constant ice mantle thickness places most of the ice on the small grains. For a given mantle thickness, this has the result of increasing the total amount of ice needed to fit the width of a given $3 \mu \mathrm{m}$ profile, in turn using up much more than the cosmically available oxygen. Smith et al. (1993) discuss this problem in terms of modeling $3 \mu \mathrm{m}$ ice profiles in the Taurus dark cloud. They find a reasonable fit to the observed profile using MRN77 cores and $0.35 \mu \mathrm{m}$ thick mantles, but the fit requires $54400 \%$ of the cosmically available oxygen. To get around this problem, Smith et al. (1993) suggest that accretion of molecular mantles in dense clouds could change the mantle thickness distribution, such that the mantle thickness varies as

$$
a_{m}=\text { const } \times a_{c}^{\beta},
$$

where $\beta$ is a positive value and the constant is a normalization factor equal to $a_{m(\max )} / a_{c(\max )}^{\beta}$. Defining the mantle thickness in this way reduces the amount of ice on the small grains, thereby reducing the total amount of ice required to fit the profile, thus keeping within the oxygen abundance constraints. Another method explored by Smith et al. was the use of a mixture of bare (no ice mantles) and mantled grains in which only a small fraction of the total grain population has ice mantles (of constant thickness). This method yielded similar results, in terms of oxygen depletion and profile fit, as the use of the mantle size distribution. However, this model required $\ll 1 \%$ of the grains to contain mantles. Such a small population of mantled grains would be difficult to explain for the Taurus or the GC molecular clouds, in which grain mantles are thought to be well pro- tected because of the cold quiescent conditions (Moneti et al. 2001a; Whittet et al. 1985, 1988). For this reason, and in order to obey the requirement that less than $100 \%$ of the available oxygen should be depleted onto the mantles, we interpret the ice profile in terms of MRN77 cores (eq. [1]) with ice mantles following the size distribution described by equation (2).

Our ice model is constructed using optical constants for pure $\mathrm{H}_{2} \mathrm{O}$ ice at $10 \mathrm{~K}, 40 \mathrm{~K}$, and $80 \mathrm{~K}$ from Hudgins et al. (1993). We allowed the maximum mantle thickness to range between $0.3-0.85 \mu \mathrm{m}$ in our model. We then used a $\chi^{2}$ fitting routine to determine the best value of $\beta$ and the maximum mantle thickness for each temperature ice. Figure 7 shows the results of our models. The best fit to the (mean) ice feature was obtained with $\beta=11$ and a maximum mantle thickness between $0.75-0.85 \mu \mathrm{m}$ for $10 \mathrm{~K} \mathrm{H}_{2} \mathrm{O}$ ice. The maximum mantle thickness is spectroscopically constrained by the astronomical profile between about 3.05-3.2 $\mu \mathrm{m}$. Mantles thicker than $0.85 \mu \mathrm{m}$ cause this segment of the model profile to be too deep compared to the mean GC ice profile, while thinner mantles do not have enough absorption in this region. However, even the thickest mantle $(0.85 \mu \mathrm{m})$ cannot
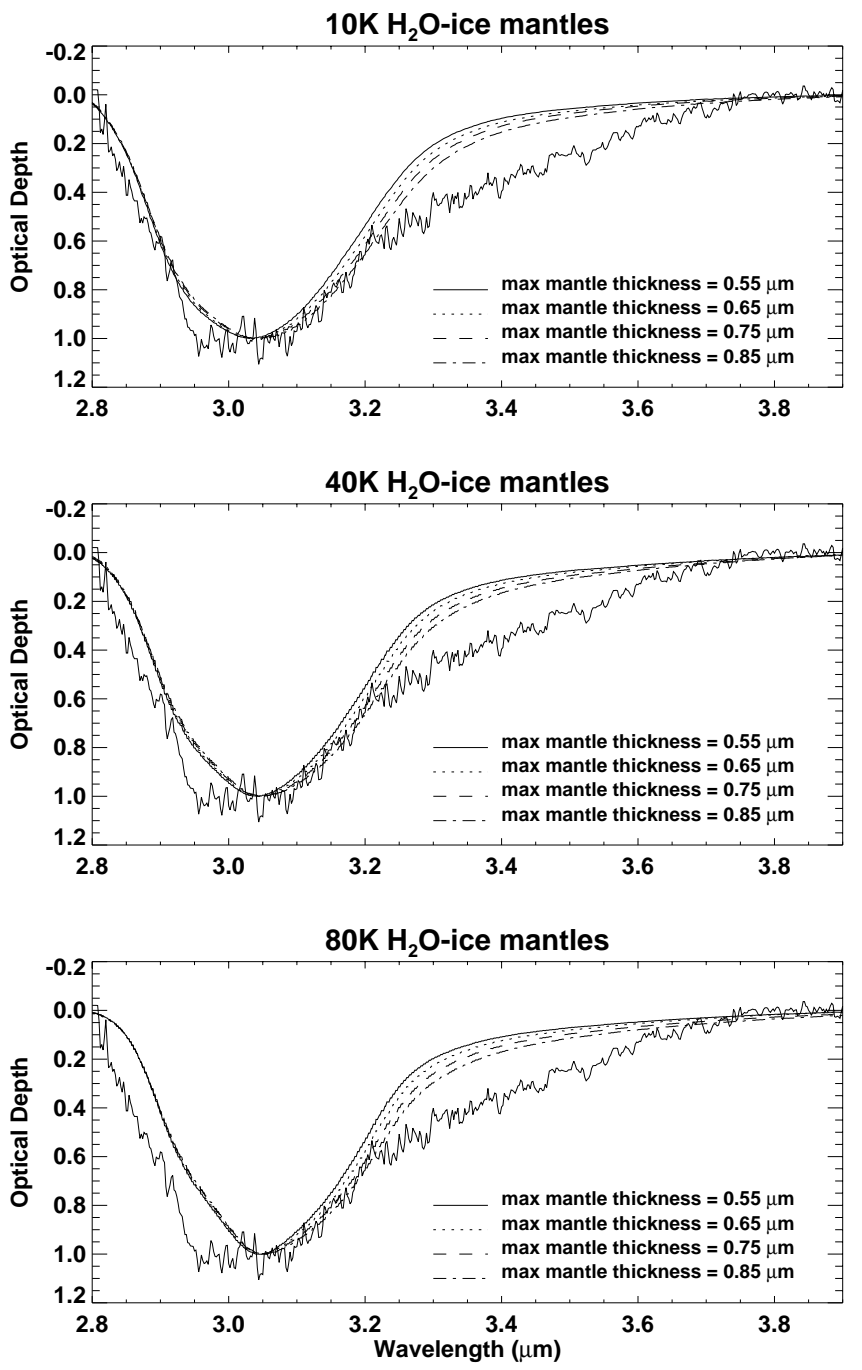

FIG. 7.-Mean GC ice feature compared with 10,40 , and $80 \mathrm{~K}$ ice models (optical constants from Hudgins et al. 1993) and maximum mantle thickness between 0.55 and $0.85 \mu \mathrm{m}$. Reasonable fits are obtained using $10 \mathrm{~K}$ $\mathrm{H}_{2} \mathrm{O}$ ice with maximum mantle thickness between 0.75 and $0.85 \mu \mathrm{m}$. 
fit the entire long-wavelength wing. Warm ice mixtures $(T \geq 40 \mathrm{~K})$ fail to fit the short side of the profile, whereas the effect of temperature on the long-wavelength wing is minimal (for the temperature range shown). Our fits thus constrain the ice temperature to be between 10 and $40 \mathrm{~K}$, consistent with previous results (Chiar et al. 2000; Moneti et al. 2001a). All the models result in residual absorption at 2.95 and $3.2-3.6 \mu \mathrm{m}$.

Another constraint on the maximum mantle thickness is the amount of cosmically available oxygen available to be incorporated into icy mantles (e.g., Pendleton, Tielens, \& Werner 1990). To check the feasibility of our result, we calculated the amount of oxygen required in pure $\mathrm{H}_{2} \mathrm{O}$ ice mantles to fit the Galactic center $3 \mu \mathrm{m}$ ice profile. Following Pendleton et al. (1990), the core volume can be written in terms of the initial core materials,

$$
V_{i}=\frac{\% \mathrm{C} m_{\mathrm{gr}} A_{\mathrm{C}}}{\rho_{\mathrm{gr}}}+\frac{\% \mathrm{Si} m_{\mathrm{sil}} A_{\mathrm{Si}}}{\rho_{\mathrm{sil}}}
$$

The percent of cosmically available carbon ( Draine (2001) as $70 \%$ and $100 \%$, respectively. Cosmic abundances of carbon and silicon are $A_{\mathrm{C}}=3.31 \times 10^{-4} \quad \mathrm{H}^{-1}$ and $A_{\mathrm{Si}}=3.55 \times 10^{-5} \mathrm{H}^{-1}$ (Grevesse \& Sauval 1998). The mass of graphite is taken to be equal to the mass of carbon $\left(m_{\mathrm{gr}}=2.00 \times 10^{-23} \mathrm{~g}\right)$ and the mass of silicate is based on $\mathrm{MgFeSiO}_{4}\left(m_{\text {sil }}=2.87 \times 10^{-22} \mathrm{~g}\right)$. The densities of graphite and silicate are $\rho_{\mathrm{gr}}=2.24 \mathrm{~g} \mathrm{~cm}^{-3}$ and $\rho_{\mathrm{sil}}=3.5 \mathrm{~g} \mathrm{~cm}^{-3}$ (Weingartner \& Draine 2001). The final grain volume with ice mantle is then

$$
V_{f}=V_{i}+\frac{\% \mathrm{O}_{\mathrm{H}_{2} \mathrm{O}} A_{\mathrm{O}}}{\rho_{\mathrm{H}_{2} \mathrm{O}}},
$$

where the mass of water $\left(m_{\mathrm{H}_{2} \mathrm{O}}\right)=2.98 \times 10^{-23} \mathrm{~g}$, the cosmic abundance of oxygen is $A_{\mathrm{O}}=6.67 \times 10^{-4} \mathrm{H}^{-1}$ (Grevesse \& Sauval 1998), and the density of water is $1 \mathrm{~g} \mathrm{~cm}^{-3}$.

In terms of the MRN77 core size distribution, the initial volume is written as

$$
\begin{aligned}
v_{i} & =\int_{a_{\min }}^{a_{\max }} \frac{4}{3} \pi n\left(a_{c}\right) a_{c}^{3} d a_{c} \\
& =\int_{a_{\min }}^{a_{\max }} \frac{4}{3} \pi n_{\mathrm{H}}\left(A_{\mathrm{sil}}+A_{\mathrm{gr}}\right) a_{c}^{3} a_{c}^{-3.5} d a_{c} .
\end{aligned}
$$

The final grain volume in terms of the MRN77 core size distribution and the Smith et al. (1993) mantle thickness distribution is

$$
\begin{aligned}
v_{f} & =\int_{a_{\min }}^{a_{\max }} \frac{4}{3} \pi n\left(a_{c}\right)\left(a_{c}+a_{m}\right)^{3} d a_{c} \\
& =\int_{a_{\min }}^{a_{\max }} \frac{4}{3} \pi n_{\mathrm{H}}\left(A_{\mathrm{sil}}+A_{\mathrm{gr}}\right)\left(a_{c}+\text { const } \times a_{c}^{\beta}\right)^{3} a_{c}^{-3.5} d a_{c} .
\end{aligned}
$$

We then set the ratios $V_{i} / V_{f}$ and $v_{i} / v_{f}$ equal to each other and solve for the oxygen depletion $(\% \mathrm{O})$. For a maximum mantle thickness between 0.55 and $0.85 \mu \mathrm{m}, 35 \%-85 \%$ of the cosmically available oxygen is required, assuming that all the grain cores along the line of sight are mantled. For the GC, it has been estimated that one-third to one-half of the visual extinction along the line of sight is due to molecular cloud material (Whittet et al. 1997). Thus, the actual $\mathrm{O}$ depletion may be lower than estimated by this simple calculation.
Although our model stays within abundance constrains, we find that the GC ice profile is not well reproduced by simple pure $\mathrm{H}_{2} \mathrm{O}$ ice mantles. It is unclear what causes the residual absorption at $2.95 \mu \mathrm{m}$ and 3.2-3.6 $\mu \mathrm{m}$. Ammonia ice may explain the $2.95 \mu \mathrm{m}$ residual ( $\S \S 1$ and 5.1) and part of the long-wavelength wing (e.g., Dartois \& d'Hendecourt 2001). Residual absorption between 3.2 and $3.6 \mu \mathrm{m}$ is observed in the ice profile of local dense clouds; however, a distinct absorption feature at $2.95 \mu \mathrm{m}$ is not seen (e.g., Smith et al. 1989; Whittet et al. 1996; Gibb et al. 2001). A portion of the 3.2-3.6 $\mu \mathrm{m}$ residual in dense clouds is explained by the presence of hydrocarbons in the ices, which exhibit a distinct feature at $3.47 \mu \mathrm{m}$ in many dense cloud sources (Brooke et al. 1996, 1999; Chiar et al. 1996). The remainder of the long-wavelength ice wing is unexplained by present models and laboratory data.

\subsubsection{Water Ice Column Density}

Column densities are calculated using the average ice profile determined in $\S 4.1$ (Fig. 5) to estimate the integrated area due to ices in each spectrum (excluding hydrocarbon absorption). This is an unbiased way of calculating the $\mathrm{H}_{2} \mathrm{O}$ ice column density as its infrared band strength $\left(A=2.0 \times 10^{-16} \mathrm{~cm}\right.$ molecule $\left.{ }^{-1}\right)$ is found to be only weakly dependent on ice mixture (Hagen, Tielens, \& Greenberg 1981; Gerakines et al. 1995). Column densities $\left[N=\int \tau(\nu) d \nu / A \mathrm{~cm}^{-2}\right.$, where $\nu$ is in units of $\left.\mathrm{cm}^{-1}\right]$ for $\mathrm{H}_{2} \mathrm{O}$ ice are listed in Table 2.

The optical depth of the $\mathrm{H}_{2} \mathrm{O}$ ice feature varies from 0.33 to 1.52 over a 2 pc region (see Table 2 and Figs. 1 and 3), and in fact varies on scales less than $0.5 \mathrm{pc}$ (e.g., compare $\tau_{3.0}$ for IRS 3 and IRS 7, located close to each other in projection). The variation of the dense cloud column over such small scales could be explained by the clumpy nature of the clouds. A recent study (Miyazaki \& Tsuboi 2000) shows that the typical clump size for GC molecular clouds ranges from 1.3 to $10 \mathrm{pc}$ where the minimum size is constrained by the angular resolution of the observations. Observations of the $\mathrm{CO}$ vibration-rotation band line of $\mathrm{CO}(4.64 \mu \mathrm{m})$ also show variations on scales of tenths of an arcsecond (Geballe et al. 1989), further evidence for the inhomogeneous nature of the clouds. In addition, we note that IRS 8 and IRS 19, which are along the line of sight toward the circumnuclear ring, and IRS 7, which is on the edge of the ring, have the deepest ice features in the sample. This finding raises the possibility that the circumnuclear ring contributes to the ice absorption along these lines of sight.

\subsection{Carbon Monoxide and $\mathrm{X}-\mathrm{C} \equiv \mathrm{N}$}

The 4.5-5.0 $\mu \mathrm{m}$ spectrum of IRS 19 reveals the presence of a deep $4.62 \mu \mathrm{m}$ "XCN" absorption band with $\tau_{4.62}=0.6$. Evidence of an XCN feature in the Sgr A* spectrum, taken with ISO SWS, was revealed by Moneti et al. (2001a), who carefully modeled the superimposed narrow $\mathrm{CO}$ gas-phase lines. In this spectrum, the feature is much weaker with $\tau_{4.62} \sim 0.1$ (estimated from Fig. 1 in Moneti et al. 2001a). It is unlikely that the feature in the IRS 19 spectrum is associated with the background illuminating source, given that it is an $\mathrm{M}$ supergiant with no obvious association with the local molecular cloud material. Although blending of gas-phase photospheric and/or interstellar $\mathrm{CO}$ absorption lines can mimic such an absorption feature, lack of absorption longward of the $4.67 \mu \mathrm{m}$ solid 


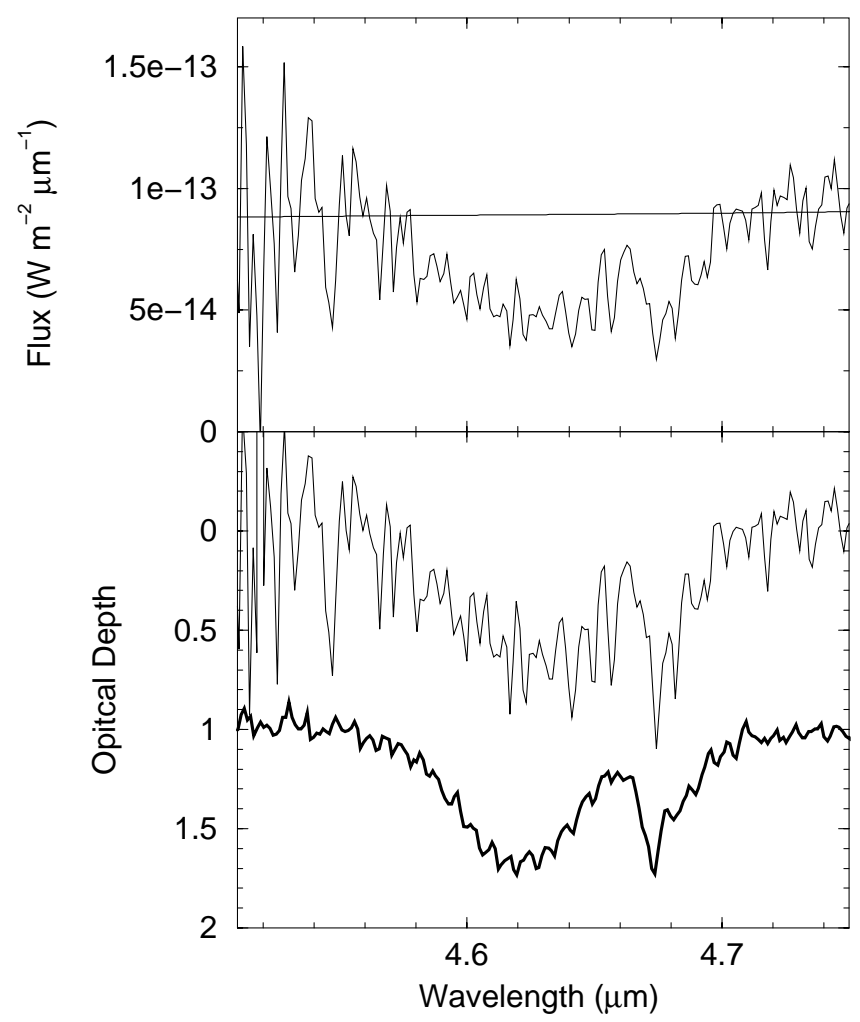

FIG. 8.-Top: Flux spectrum of IRS 19 from 4.51 to $4.75 \mu \mathrm{m}$ shown with polynomial continuum fit (straight line). Bottom: Optical depth spectrum showing deep XCN absorption at $4.62 \mu \mathrm{m}$ and solid $\mathrm{CO}$ absorption at 4.67 $\mu \mathrm{m}$ (thin line). The optical depth spectrum of W33A is shown for comparison offset and scaled to $\tau_{4.62}=0.6$ (thick line; spectrum from Chrysostomou et al. 1996).

$\mathrm{CO}$ feature (where the $\mathrm{P}$ branch lines of gaseous $\mathrm{CO}$ would absorb) rule out this possibility. The carrier of the $4.62 \mu \mathrm{m}$ feature is thought to be a product of energetic processing of interstellar ices (Pendleton et al. 1999 and references therein). Although analog materials produced in the laboratory are quite hardy and refractory (Palumbo et al. 2000a), suggesting that the interstellar $4.62 \mu \mathrm{m}$ band carrier might survive the conditions of the diffuse ISM, the feature is not seen in the spectrum of diffuse interstellar dust to current sensitivity limits (Whittet et al. 2001a). If the limit $\tau_{4.62} / A_{V}<0.0015$ determined for Cyg OB2\#12 (Whittet et al. 2001a) is applied to the GC, optical depths $\tau_{4.62}<0.05$ are predicted, assuming that all the dust responsible for extinction along the line of sight contributes to the feature. This is an order of magnitude less than the strength of the feature detected in IRS 19. We therefore conclude that the $4.62 \mu \mathrm{m} \mathrm{XCN}$ feature in the spectrum of IRS 19 most likely arises in a dense molecular cloud along the line of sight, rather than in the diffuse ISM.

In the solar neighborhood, $\mathrm{XCN}$ is seen only in the spectra of certain embedded protostellar sources, of which W33A is the prototypical example (Fig. 8); it is not seen in the quiescent dark-cloud medium (Whittet et al. 2001a and references therein). The carrier, which is yet to be securely identified, appears to be formed when icy coated grains are subjected to ultraviolet photolysis and/or energetic particle bombardment (Pendleton et al. 1999). Of the various candidates, those with cyanate $(-\mathrm{O}-\mathrm{C} \equiv \mathrm{N})$ structure are most consistent with the observations in terms of their spectroscopic properties (Grim \& Greenberg 1987; Schutte \&
Greenberg 1997; Pendleton et al. 1999; Bernstein, Sandford, \& Allamandola 2000; Palumbo et al. 2000a). For IRS 19, we estimate the column density to be $N=3 \times 10^{17} \mathrm{~cm}^{2}$, assuming an absorption band strength of $A=5 \times 10^{-17} \mathrm{~cm}^{-1}$ (the "average" band strength of presently acceptable XCN candidates; see Whittet et al. 2001a for details.).

There has been much recent interest in the possibility that cosmic synthesis of $\mathrm{CN}$-bearing species may lead to the inclusion of such molecules in protoplanetary disks, and these may in turn become an important component of prebiotic matter in protoplanetary disks (Tegler et al. 1995; Pendleton et al. 1999; Whittet et al. 2001b). The presence of the $4.62 \mu \mathrm{m}$ feature in the spectrum of a GC source offers an additional setting in which this feature can be studied. It may arise by the serendipitous presence of a young stellar object embedded in a molecular cloud along the line of sight to IRS 19 , or it might conceivably have a more exotic origin, perhaps arising in the circumnuclear ring of the GC itself.

Gas-phase interstellar CO has been previously detected toward several of the IR sources discussed here (IRS $1 \mathrm{~W}, 3$, 6E, 7, and 8; Geballe et al. 1989). Gas-phase CO lines are also present in the ISO SWS spectra centered on Sgr A* and the Quintuplet sources (Lutz et al. 1996; Chiar et al. 2000), and are well reproduced by a cold gas model (Moneti et al. 2001a). Detection of solid CO at $4.67 \mu \mathrm{m}$ has proved to be more difficult. It was previously detected toward IRS 12 and also revealed in the ISO SWS Sgr A* spectrum via modeling of the gas-phase $\mathrm{CO}$ lines (Moneti et al. 2001a). Figure 8 shows the first detection of the solid CO toward IRS 19. Analysis of the IRS 19 spectrum results in $\mathrm{CO} / \mathrm{H}_{2} \mathrm{O}=11 \%$, consistent with previous estimates $\left(\mathrm{CO} / \mathrm{H}_{2} \mathrm{O}<12 \%\right.$; Chiar et al. 2000; McFadzean et al. 1989).

\section{HYDROCARBONS}

Our deconvolution of the ice and hydrocarbon profiles (§ 4.1) reveals residual absorption at $\sim 3.3$ and $3.4 \mu \mathrm{m}$. The latter is the well-studied aliphatic hydrocarbon feature that is, to date, observed in diffuse ISM sight lines with visual extinction greater than 4 mag (Sandford et al. 1991; Pendleton et al. 1994). Previous derivations of the $3.4 \mu \mathrm{m}$ profile typically employed a local continuum that removed the new $3.3 \mu \mathrm{m}$ absorption feature. Hydrogenated amorphous carbon (HAC) has been found to provide an excellent representation (in both position and relative intensity) of the aliphatic hydrocarbon absorption features at 3.4, 6.85, and $7.25 \mu \mathrm{m}$ observed in the diffuse ISM (Chiar et al. 2000; Pendleton \& Allamandola 2002). Excluding IRS 7 and IRS 12 , we find that the strongest hydrocarbon band, toward IRS 3 , is 1.7 times that in IRS $1 \mathrm{~W}$, which displays the weakest $3.4 \mu \mathrm{m}$ band. Variation in depth of the $3.4 \mu \mathrm{m}$ band likely reflects distribution changes in the diffuse ISM and thus corresponds to largely foreground material. Cotera et al. (2000) showed that on arcminute scales, the total extinction in this region varies by around $5 \mathrm{mag}$ (in 30) over $1^{\prime}$ (although their study centered on the region around Sgr A East). However, our results imply changes in the foreground diffuse ISM extinction greater than $50 \%$ across the small field studied here.

To determine the nature of the additional absorption centered around $3.3 \mu \mathrm{m}$, we plot its depth against those of the $\mathrm{H}_{2} \mathrm{O}$ ice feature (Fig. 9, top) and the $3.4 \mu \mathrm{m}$ aliphatic hydrocarbon feature (Fig. 9, bottom). No trend is apparent in the $\tau_{3.0}$ versus $\tau_{3.3}$ plot; thus, it is unlikely that the $3.3 \mu \mathrm{m}$ fea- 

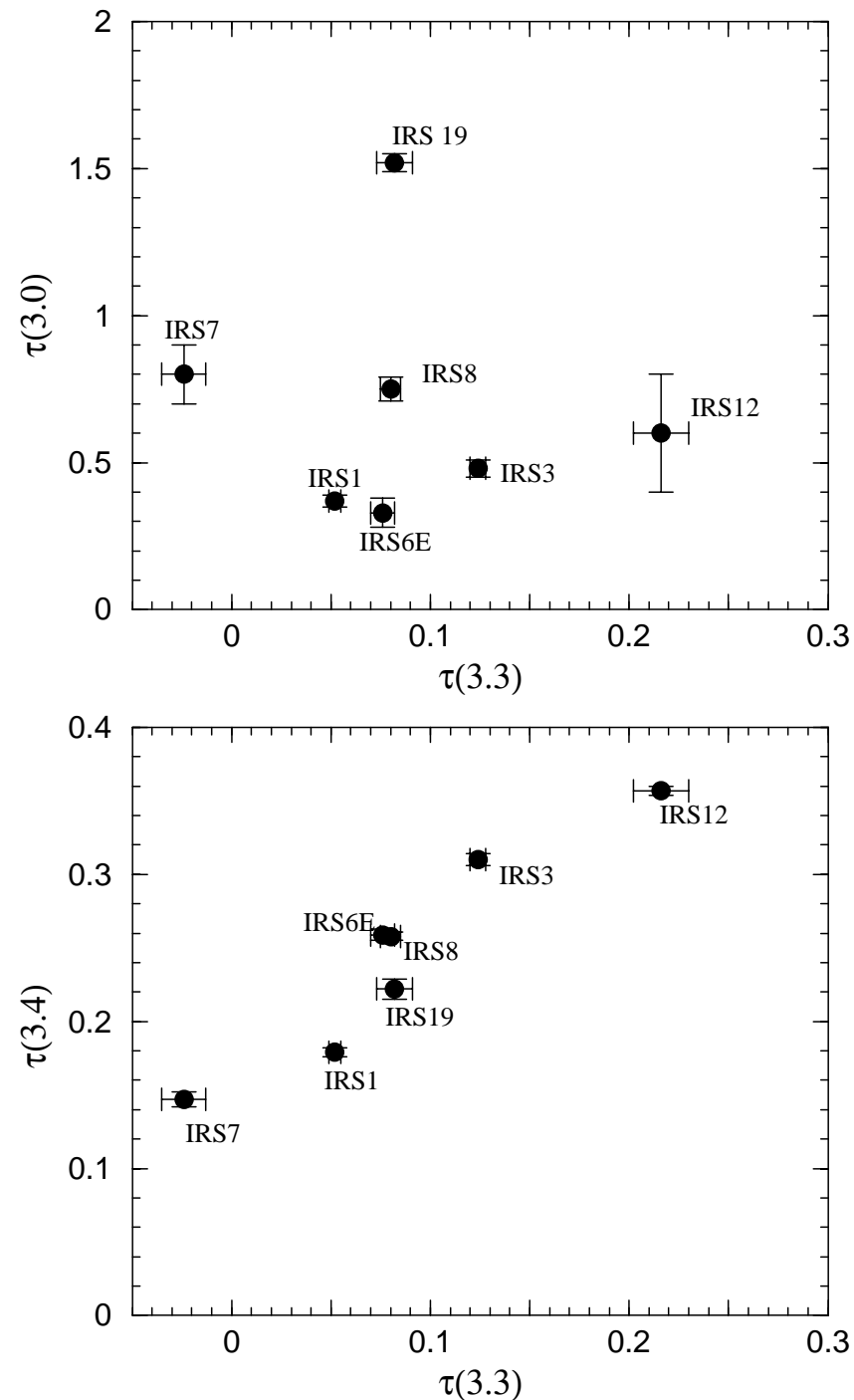

FIG. 9.-Top: Water ice optical depth vs. $3.3 \mu \mathrm{m}$ absorption feature depth for GC sources. No trend is apparent between the ice feature and the $3.3 \mu \mathrm{m}$ absorption. Bottom: Optical depth of the $3.4 \mu \mathrm{m}$ hydrocarbon absorption feature vs. the depth of the $3.3 \mu \mathrm{m}$ absorption. The correlation between these absorption features is suggestive that the $3.3 \mu \mathrm{m}$ absorption is carried by dust in the diffuse ISM rather than the molecular cloud material. IRS 7 has a negative $\tau_{3,3}$ value since its ice profile is more narrow than the mean used to determine the excess hydrocarbon absorption. See text for details on determination of these depths

ture is carried by ices in the dense cloud material. Instead, the correlation between $\tau_{3.4}$ versus $\tau_{3.3}$ suggests that the carrier of the $3.3 \mu \mathrm{m}$ absorption is part of the diffuse ISM. Since the correlation intercepts the $\tau_{3.4}$ axis, the $3.3 \mu \mathrm{m}$ absorption probably contributes to the depth of the $3.4 \mu \mathrm{m}$ profile, and its central wavelength may well be longward of $3.3 \mu \mathrm{m}$. We deduce that the " $3.3 \mu \mathrm{m}$ " profile is quite broad, at least 100 $\mathrm{cm}^{-1}(0.1 \mu \mathrm{m})$ in width. Pendleton et al. (1994) discuss the possible presence of narrow (FWHM $\sim 50 \mathrm{~cm}^{-1}$ ) absorption around $3.3 \mu \mathrm{m}$ in the spectra of IRS 6E, IRS 7, and IRS 3 ; however, the presence of the Pfund $\delta$ hydrogen recombination line at $3.29 \mu \mathrm{m}$ in the comparison star spectra made that detection tentative at best. We do not see evidence for this discrete feature in any of the spectra presented here.

It is tempting to attribute the absorption feature to polycyclic aromatic hydrocarbons (PAHs), which have features around $3.3 \mu \mathrm{m}$. Typical astronomical $3.3 \mu \mathrm{m}$ PAH emission features have widths $\sim 30 \mathrm{~cm}^{-1}$ (Sellgren 2001 and references therein). Although temperature effects can lead to broadening of the profile because of higher $J$ levels being populated at higher temperature (Joblin et al. 1995), a width of $100 \mathrm{~cm}^{-1}$ would imply temperatures greater than $1000 \mathrm{~K}$, much too high for the diffuse ISM, where the temperature of dust grains is constrained to $10-25 \mathrm{~K}$ (Mathis et al. 1983). Brooke et al. (1999) observe a similarly broad $\left(\sim 74 \mathrm{~cm}^{-1}\right)$ absorption feature at $3.25 \mu \mathrm{m}$ in several lines of sight toward dense clouds, where the feature is attributed to cold aromatic hydrocarbons. Chiar et al. (2000) observed absorption centered at $3.28 \mu \mathrm{m}$ toward the GC Quintuplet source GCS 3..$^{5}$ Its width $\left(25 \mathrm{~cm}^{-1}\right)$ and central wavelength are more similar to the ubiquitous emission features observed throughout the Galaxy. This relatively narrow feature does not appear to be present in the spectra presented here of the central cluster sources. It is uncertain which effects (temperature, composition, size distribution, ionization state) cause the differences in width and central wavelength of these absorption/emission features observed in different environments (see Sellgren 2001), but some combination of PAHs affected by these factors likely accounts for the broad " 3.3 $\mu \mathrm{m}$ " absorption feature toward the GC.

Mixtures of hydrogenated amorphous carbon (HAC) and PAHs, which exhibit features at both 3.3 and $3.4 \mu \mathrm{m}$, have been suggested as candidates for the unidentified infrared bands $(3.3,6.2,7.7,8.6,11.3$, and $12.7 \mu \mathrm{m})$ (e.g., Allamandola, Tielens, \& Barker 1985; Borghesi, Bussoletti, \& Colangeli 1987; Blanco, Bussoletti, \& Colangeli 1988). While most laboratory analogs possess features at longer wavelengths that are not seen in astronomical spectra, Pendleton \& Allamandola (2002) conclude the organic refractory component of the diffuse ISM is largely hydrocarbon in nature, with the carbon distributed between the aromatic and aliphatic forms. Thus, further laboratory studies are needed on these materials to determine if they are suitable candidates for the hydrocarbon absorption features discussed here.

\section{CONCLUSIONS}

Good-quality spectra of a sample of GC sight lines with different relative strengths of the ice and hydrocarbon absorption bands permit the two components to be isolated and their mean spectral profiles to be generated. Absorption in both the ice and hydrocarbon bands varies considerably in strength across the GC field, although the band profiles remain essentially constant. Variability of the depth of the ice band is expected because of the patchy nature of the molecular cloud distribution across the field. The depth of the ice band varies by a factor of almost 5 , and the deepest ice feature occurs along the line of sight toward IRS 19 with a band twice as deep $\left(\tau_{3.0}=1.52\right)$ as the next deepest feature (IRS 8, $\tau_{3.0}=0.75$ ). The three ice bands of greatest optical depth occur toward sources that lie along the line of sight toward the circumnuclear ring (IRS 19, IRS 8, and IRS 7,

\footnotetext{
${ }^{5}$ The unidentified infrared emission bands at $6.2,7.7,8.6$, and $11.3 \mu \mathrm{m}$ are detected in the spectrum of the background along the line of sight toward the Quintuplet sources (Moneti et al. 2001b). Given that the emission is uniform over the Quintuplet region, it is conceivable that it could extend to the GC itself. However, background emission would be removed in our nodded observations.
} 
which lies at the inner edge of the ring), suggesting that the circumnuclear ring may be responsible for some of the absorption.

Variation in the hydrocarbon absorption strength is less easily explained than that in the ice band and reflects changes in the distribution of the foreground diffuse ISM. Our results imply changes in the diffuse ISM extinction by greater than $50 \%$. To make further progress on the relationships between these dust (and ice) features and their spatial origins requires a much larger sample of objects, distributed across the entire central field including the circumnuclear ring and central cluster. While this is not currently feasible in spectroscopy, imaging in narrowband filters covering the $3 \mu \mathrm{m}$ window should enable the ice, 3.28 , and $3.4 \mu \mathrm{m}$ feature strengths to be broadly quantified over the required field. Such a program is currently being pursued by the authors at UKIRT.

Our deconvolution of absorption due to dense cloud and diffuse ISM components clearly reveals an additional absorption band centered near $3.3 \mu \mathrm{m}$. Based on the correlation of this feature with the $3.4 \mu \mathrm{m}$ aliphatic hydrocarbon feature, we deduce that it is not due to ice absorption, but is suggestive of absorption due to PAHs. However, its width is much broader than the observed unidentified infrared emission feature (at $3.3 \mu \mathrm{m}$ ) and the absorption feature observed toward the GC Quintuplet source GCS3. While a similarly broad feature is observed in some dense cloud sight lines, it occurs shortward of the feature deduced for the GC. The width and central wavelength of the " $3.3 \mu \mathrm{m}$ " feature could be indicative of various environmental effects on a combination of PAHs.

Water ice absorption produces the majority of the integrated absorption in the $3 \mu \mathrm{m}$ spectral region in these lines of sight. Isolating the ice band, as described in $\S 4.1$, produces a profile that cannot be entirely fitted by pure $\mathrm{H}_{2} \mathrm{O}$ (see also Chiar et al. 2000 for discussion of fits to the GC spectrum using laboratory data). We have attempted to model the ice feature using a Mie scattering code for the ice-coated grains assuming a core size distribution $n\left(a_{c}\right) \propto a_{c}^{-3.5}$ and a mantle thickness distribution $a_{m} \propto a_{c}^{\beta}$. The (so-called) best fit to the GC ice profile requires quite thick mantles and a significant portion of the cosmically available O. Even then, a long-wavelength absorption "wing," from 3.2 to $3.6 \mu \mathrm{m}$, is apparent in addition to the hydrocarbon absorption bands. Furthermore, absorption on the short-wavelength wing of the band is present at $2.95 \mu \mathrm{m}$, suggesting the presence of $\mathrm{NH}_{3}$ in ices. In addition, $\mathrm{NH}_{3}$ could be responsible for some of the absorption in the ice wing. To make further progress on a model that completely describes the ice profile requires optical constants of ice mixtures such as $\mathrm{H}_{2} \mathrm{O}: \mathrm{NH}_{3}$ with relative abundances constrained by current data (e.g., $\mathrm{H}_{2} \mathrm{O}: \mathrm{NH}_{3} \approx 100: 10$ ).

We detect the $4.62 \mu \mathrm{m} \mathrm{XCN}$ feature in the line of sight toward IRS 19, providing evidence for $\mathrm{CN}$-bearing solids in the molecular clouds toward the GC. The depth of the XCN feature $\left(\tau_{4.62}=0.6\right)$ indicates that it is not part of the diffuse ISM along the line of sight. It is in the range of detections toward young stellar objects, where $\tau_{4.62}$ ranges from 0.05 to 1.5 (Palumbo et al. 2000b and references therein). We also detect solid $\mathrm{CO}$ at $4.67 \mu \mathrm{m}$ in the IRS 19 spectrum. The abundance of solid $\mathrm{CO}$ relative to $\mathrm{H}_{2} \mathrm{O}$ is in agreement with the previous detection toward IRS 12 (McFadzean et al. 1989) and previous limits (Chiar et al. 2000).

The United Kingdom Infrared Telescope is operated by the Joint Astronomy Centre on behalf of the UK Particle Physics and Astronomy Research Council. The authors wish to thank George Hassel at Rensselaer for developing and running the code to model the ice feature. Data for IRS 7 were obtained via the UKIRT Archive located at the UK Astronomy Data Centre in Cambridge. J. E. C. is supported by NASA's Long-Term Space Astrophysics program under grant 399-20-61-02. Y. J. P. thanks the NASA exobiology program (344-38-12-09) for support of her participation in this work. D. C. B. W. is funded by NASA through the Exobiology and Long-Term Space Astrophysics programs (grants NAG5-7598 and NAG5-7884). J. E. C. wishes to thank the Joint Astronomy Centre for their hospitality during her sabbatical visit when much of this work was accomplished. This research has made use of the SIMBAD database, operated at CDS, Strasbourg, France. The authors acknowledge the data analysis software provided by the Starlink Project, which is run by CCLRC on behalf of PPARC. The authors wish to thank the referee, Andrea Moneti, for his comments, which helped improve the manuscript.

\section{REFERENCES}

Adamson, A. J., Whittet, D. C. B., Chrysostomou, A., Hough, J. H., Aitken, D. K., Wright, G. S., \& Roche, P. F. 1999, ApJ, 512, 224

Allamandola, L. J., Sandford, S. A., Tielens, A. G. G. M., \& Herbst, T. M. 1992, ApJ, 399, 134

Allamandola, L. J., Tielens, A. G. G. M., \& Barker, J. R. 1985, ApJ, 290, L25

Bailey, J., Hough, J. H., \& Axon, D. J. 1984, MNRAS, 208, 661

Becklin, E. E., Matthews, K., Neugebauer, G., \& Willner, S. P. 1978, ApJ, 219, 121

Becklin, E. E., \& Neugebauer, G. 1975, ApJ, 200, L71

Bernstein, M. P., Sandford, S. A. \& Allamandola, L. J. 2000, ApJ, 542, 894

Blanco, A., Bussoletti, E., \& Colangeli, L. 1988, ApJ, 334, 875

Blum, R. D., Depoy, D. L., \& Sellgren, K. 1995, ApJ, 441, 603

Blum, R. D., Sellgren, K., \& Depoy, D. L 1996, ApJ, 470, 864

Bohren, C. F., \& Huffman, D. R. 1983, Absorption and Scattering of Light by Small Particles (New York: Wiley)

Borghesi, A., Bussoletti, E., \& Colangeli, L. 1987, ApJ, 314, 422

Brooke, T. Y.., Sellgren, K., \& Geballe, T. R. 1999, ApJ, 517, 883

Brooke, T. Y., Sellgren, K., \& Smith, R. G. 1996, ApJ, 459, 209

Butchart, I., McFadzean, A. D., Whittet, D. C. B., Geballe, T. R., \& Greenberg, J. M. 1986, A\&A, 154, L5

Chan, K., et al. 1997, ApJ, 483, 798

Chiar, J. E., Adamson, A. J., \& Whittet, D. C. B. 1996, ApJ, 472, 665

Chiar, J. E., Pendleton, Y. J., Geballe, T. G., \& Tielens, A. G. G. M. 1998, ApJ, 507, 281
Chiar, J. E., \& Tielens, A. G. G. M. 2001, ApJ, 550, L207

Chiar, J. E., et al. 2000, ApJ, 537, 749

Chrysostomou, A., Hough, J. H., Whittet, D. C. B., Aitken, D. K., Roche, P. F., \& Lazarian, A. 1996, ApJ, 465, L61

Cotera, A. S., Simpson, J. P., Erickson, E. F., Colgan, S. W. J., Burton, M. G., \& Allen, D. A. 2000, ApJS, 129, 123

Dartois, E., \& d'Hendecourt, L. 2001, A\&A, 365, 144

Dartois, E., Schutte, W., Geballe, T. R., Demyk, K., Ehrenfreund, P., \& d'Hendecourt, L. 1999, A\&A, 342, L32

de Graauw, T., et al. 1996, A\&A, 315, L345

Draine, B. T., \& Lee, H. M. 1984, ApJ, 285, 89

Geballe, T. R., Baas, F., \& Wade, R. 1989, A\&A, 208, 255

Gerakines, P. A., Schutte, W. A., Greenberg, J. M., \& van Dishoeck, E. F. 1995, A\&A, 296, 810

Gerakines, P. A., et al. 1999, ApJ, 522, 357

Gibb, E. L., Whittet, D. C. B., \& Chiar, J. E. 2001, ApJ, 558, 702

Grevesse, N., \& Sauval, A. J. 1998, Space Sci. Rev., 85, 161

Grim, R. J. A., Baas, F., Greenberg, J. M., Geballe, T. R., \& Schutte, W. 1991, A\&A, 243, 473

Grim, R. J. A., \& Greenberg, J. M. 1987, ApJ, 321, L91

Güsten, R., Genzel, R., Wright, M. C. H., Jaffe, D. T., Stutzki, J., \& Harris, A. 1987, in AIP Conf. Proc. 155, The Galactic Center, ed. D. C. Backer (New York: AIP), 103

Hagen, W., Tielens, A. G. G. M., \& Greenberg, J. M. 1981, Chem. Phys., 56,367 
Herbst, T. M., Beckwith, S. V. W., Forrest, W. J., \& Pipher, J. L. 1993, AJ, 105,956

Hinkle, K., Wallace, L., \& Livingston, W. 1995, PASP, 107, 1042

Holbrook, J. C., \& Temi, P. 1998, ApJ, 496, 280

Hudgins, D. M., Sandford, S. A., Allamandola, L. J., \& Tielens, A. G. G. M. 1993, ApJS, 86, 713

Joblin, C., Boissel, P., Leger, A., d'Hendecourt, L., \& Defourneau, D. 1995, A\&A, 299, 835

Krabbe, A., et al. 1995, ApJ, 447, L95

Laor, A., \& Draine, B. T. 1993, ApJ, 402, 441

Latvakoski, H. M., Stacey, G. J., Gull, G. E., \& Hayward, T. L. 1999, ApJ, 511,761

Lebofsky, M. J. 1979, AJ, 84, 324

Lebofsky, M. J., Rieke, G. H., \& Tokunaga, A. T. 1982, ApJ, 263, 736

Lutz, D., et al. 1996, A\&A, 315, L269

Martin, P. G., \& Whittet, D. C. B. 1990, ApJ, 357, 113

Mathis, J. S., Mezger, P. G., \& Panagia, N. 1983, A\&A, 128, 212

Mathis, J. S., Rumpl, W., \& Nordsieck, K. H. 1977, ApJ, 217, 425 (MRN77)

McFadzean, A. D., Whittet, D. C. B., Bode, M. F., Adamson, A. J., \& Longmore, A. J. 1989, MNRAS, 241, 873

Miyazaki, A., \& Tsuboi, M. 2000, ApJ, 536, 357

Moneti, A., Cernicharo, J., \& Pardo, J. R. $2001 \mathrm{a}$, ApJ, 549, L203

Moneti, A., Stolovy, S., Blommaert, J. A. D. L., Figer, D. F., \& Najarro, F. 2001b, A\&A, 366, 106

Palumbo, M. E., Pendleton, Y. J., \& Strazzulla, G. 2000a, ApJ, 542, 890

Palumbo, M. E., Strazzulla, G., Pendleton, Y. J., \& Tielens, A. G. G. M. 2000b, ApJ, 534, 801

Pendleton, Y. J., \& Allamandola, L. J. 2002, ApJS, 138, 75

Pendleton, Y. J., Sandford, S. A., Allamandola, L. J., Tielens, A. G. G. M., \& Sellgren, K. 1994, ApJ, 437, 683

Pendleton, Y. J., Tielens, A. G. G. M., Tokunaga, A. T., \& Bernstein, M. P. 1999, ApJ, 513, 294

Pendleton, Y. J., Tielens, A. G. G. M., \& Werner, M. W. 1990, ApJ, 349, 107
Rieke, G. H., \& Low, F. J. 1973, ApJ, 184, 415

Rieke, G. H., Rieke, M. J., \& Paul, A. E. 1989, ApJ, 336, 752

Sanders, D. B., Scoville, N. Z., \& Solomon, P. M. 1985, ApJ, 289, 373

Sandford, S. A., Allamandola, L. J., Tielens, A., Sellgren, K., Tapia, M., \& Pendleton, Y. 1991, ApJ, 371, 607

Schutte, W. A., \& Greenberg, J. M. 1997, A\&A, 317, L43

Sellgren, K. 2001, Spectrochim. Acta, 57, 627

Sellgren, K., Brooke, T. Y., Smith, R. G., \& Geballe, T. R. 1995, ApJ, 449, L69

Simon, M., Chen, W.-P., Forrest, W. J., Garnett, J. D., Longmore, A. J., Gauer, T., \& Dixon, R. I. 1990, ApJ, 360, 95

Smith, R. G., Sellgren, K., \& Brooke, T. Y. 1993, MNRAS, 263, 749

Smith, R. G., Sellgren, K., \& Tokunaga, A. T. 1989, ApJ, 344, 413

Storey, J. W. V., \& Allen, D. A. 1983, MNRAS, 204, 1153

Tegler, S. C., Weintraub, D. A., Rettig, T. W., Pendleton, Y. J., Whittet, D. C. B., \& Kulesa, C. A. 1995, ApJ, 439, 279

Tielens, A. G. G. M., Wooden, D. H., Allamandola, L. J., Bregman, J., \& Witteborn, F. C. 1996, ApJ, 461, 210

Tollestrup, E. V., Becklin, E. E., \& Capps, R. W. 1989, AJ, 98, 204

Wada, S., Sakata, A., \& Tokunaga, A. T. 1991, ApJ, 375, L17

Weingartner, J. C., \& Draine, B. T. 2001, ApJ, 548, 296

Whittet, D. C. B., Bode, M. F., Longmore, A. J., Adamson, A. J., McFadzean, A. D., Aitken, D. K., \& Roche, P. F. 1988, MNRAS, 233 321

Whittet, D. C. B., Gibb, E. L., \& Nummelin, A. 2001a, Origins Life Evol. Biosphere, 31, 157

Whittet, D. C. B., McFadzean, A. D., \& Longmore, A. J. 1985, MNRAS, $216,45 \mathrm{P}$

Whittet, D. C. B., Pendleton, Y. J., Gibb, E., Boogert, A. C. A., Chiar, J. E., \& Nummelin, A. 2001b, ApJ, 550, 793

Whittet, D. C. B., et al. 1996, ApJ, 458, 363

.1997, ApJ, 490, 729

Wollman, E. R., Smith, H. A., \& Larson, H. P. 1982, ApJ, 258, 506 